\title{
VTLOGANL: Coding and analyzing videotaped data
}

\author{
JEFFREY B. HECHT and NICOLE K. ROBERTS \\ Illinois State University, Normal, Illinois
}

\begin{abstract}
Researchers using videotape as a primary means of data collection need flexible yet easy-to-use procedures and equipment for the time-consuming task of coding and analysis. The VTLOGANL program (for VideoTape LOGging ANaLysis) slaves a video deck to the computer system, allowing the researcher to code, in real time, video-recorded events of either a pulsed or duration nature. VTLOGANL's innovative database design frees the researcher to define up to 9,999 different codewords for each project, organizing any combination of up to 30 of those codewords for each elapsed second of logging time. Reporting features, including data export and import, allow the researcher to examine quickly questions of codeword frequency, event duration, sequencing, and overlap, according to the requirements of the research design.
\end{abstract}

Behavioral researchers employ a wide variety of research methodologies to gather and analyze data. To varying degrees, researchers observe their subjects, conduct interviews and surveys, collect and analyze physical evidence, examine patterns of physical erosion, and interact with their subjects in real situations. Numerous manual paper-and-pencil data techniques have been developed and refined over the years to help researchers systematize the often enormous task of data recording and analysis. The advent of computers, along with low-cost and easy-to-operate audio-only and audio-video recording equipment, has added to the available repertoire of methodologies. In many cases the new technology is used with existing methodologies, rather than exploiting the potential richness technology can provide. Such has been the case with data collection using video recording. The VTLOGANL (for VideoTape LOGging ANaLysis) development project, described in this article, refined a methodology and developed a computer software tool that can be used to apply systematic traditions while engaging all the flexibility, ease of use, and speed that modern technology can offer.

\section{Data-Gathering Methodologies}

One of the least expensive and easy-to-implement methods of collecting data in the field is the taking of written notes with paper and pencil. Gardner, Clements, and Rodriquez (1982) noted that the observer can be writing of one event while simultaneously being aware of

A prior version of this paper was presented at the 1994 Annual Meeting of the American Educational Research Association, New Orleans. The authors would like to thank the many reviewers who have commented on VTLOGANL and this manuscript during its development and trial testing. Correspondence should be addressed to J. B. Hecht, Technological Innovations in Educational Research Laboratory, College of Education, Illinois State University, Normal, IL 61790-5900 (e-mail: jbhecht@ilstu.edu). another, holding the latter event memory until the current writing is accomplished. Neal (1989) found, however, that notetaking relies on a consistent application of observer attention to task and detail as well as the ability to accurately recall dialogue and events that may be happening faster than one's ability to write. This often results in a reduction in recorded data and more inaccuracies in the data that are recorded.

Audio recordings improve on notetaking, capturing exact words that can be later transcribed and analyzed. Analysis of the audio record can reveal nuances of speech patterns, dialect, and intonation that often subtly shade the meaning of spoken words. Higher levels of background noise, the inability to place microphones near enough to the speakers for adequate sound reproduction, multiple and/or simultaneous speakers, and the significance of gestures and other physical behavior, however, reduce the effectiveness of audio recording as a research tool. In addition, Kieren and Munro (1985) found that nonstenographers can take up to $2 \frac{1}{2} \mathrm{~h}$ to transcribe $10 \mathrm{~min}$ of audiotape (p. 13).

Video recordings provide researchers both auditory and visual information, freeing them to engage more fully in an interview or interaction with subjects. Further, written field notes can be oriented toward the interpretation of the activity rather than its verbatim reproduction. Video also allows researchers unlimited opportunities to revisit and reexamine the observed situation. Events can be timed, accurate transcripts of speech written, settings and movement drawn and described, and hypotheses tested against a framework of reexaminable data.

The large quantity of information that can be extracted from video creates a problem, however, for social science researchers. Data overload is intensified by video because so much more detail of the event is preserved and available for analysis. Although this heightened level of detail can lead to better understanding, the sheer volume of available information can overwhelm even the most prac- 
ticed analyst (Knapp \& Harrison, 1972). As Carter and Anderson (1989) noted, researchers must determine that video is the appropriate technique for collecting the type of data required for investigating the question(s) at hand before they begin collecting data.

\section{Analyzing Video as Text}

Two techniques have emerged for analyzing videorecorded data: creating a verbatim transcript of spoken language, which often includes a descriptive narration of actions, settings, and activities; and creating a code list of words or short phrases representing a conceptual interpretation of verbal exchanges and visual observations. Our review of the research literature reveals that many researchers who use video for original data collection first transcribe the videotape and then analyze the resulting transcript using textual analytical processes (e.g., Barba \& Rubba, 1992; Lo, Wheatley, \& Smith, 1991; Scarselletta, 1987; Schotsberger, 1993). Other researchers use some form of concept coding to analyze their videotapes (e.g., Packer \& Richardson, 1989; Stallings \& Giesen, 1977; Tait, 1993). The reexpression of audio and video data into written text for classification and analysis is familiar, and therefore compelling. The analysis of text derived from a video record, however, entails the risk of disregarding the complex interrelatedness of words and actions, expression and activity (Knapp \& Harrison, 1972).

\section{Analyzing Video as Video}

Since the advent of videotape technology, researchers have sought means of avoiding this discarding of useful information by performing their analyses directly from the videotape. Early researchers were hampered by overly complicated and costly video equipment not designed for this task, as well as the lack of a coherent methodology for categorization and review. In the past few years, though, several systematic methods have emerged as potential ways to conduct an analysis of video data.

Videologger, a software package described and developed by Krauss, Morrel-Samuels, and Hochberg (1988), provided the user with a way to document specific, preordained events having multiple durations. Videologger used one of the audio tracks available on a videotape as a marker, creating an audio notation when the videographer pushed a button on the camcorder indicating that a desired event was beginning and ending. EVA, developed by Mckay (1989), allowed for annotation of the videotaped data as they are collected in the field. EVA could also be used to find tags created by the researcher, then to transcribe the audio track, and finally to view the video with the transcription running at the same time. Roschelle and Goldman (1991) developed VideoNoter, a tool that links the video record with transcripts, codes, annotations, and other secondary records of data. They described their tool as a system of progressive refinements, enabling the researcher to focus in on the most interesting or applicable parts of the data. CVideo, available since 1992 (J. Roschelle, personal communication,
November 22, 1994), provides computerized VCR controls that support general-purpose notetaking into word processing files. The latest version (since October 1993) provides VCR control services to virtually any Apple program, allowing users to link the VCR with other word processing, spreadsheet, or database software application.

Currently available software packages can be classified according to several different criteria, including (1) required computer and/or video hardware; (2) types of data management services provided (data entry, change, deletion, import and export, and reporting); (3) the kinds of coding allowed (varying from unlimited amounts of freeformat text to limited numbers of fixed-expression code numbers or words); (4) the types of events that can be easily coded (from events having short, finite durations to longer, open-ended duration events, and from events that are coded whenever they occur to events coded only at certain intervals and/or only for a set period); and (5) the capacity and speed of the database (the number of different codes that can be entered, the total number of events and/or duration that can be coded, and the speed of the database engine in retrieving previously coded data). Some packages require specialized hardware to connect the video deck to the computer or special hardware on the computer to display the data. Others, like CVideo, focus primarily on providing VCR control services, leaving the user free to choose the data management software (word processor, spreadsheet, or database) appropriate to their needs. Many of the packages are available only on the Apple computer platform, leaving MS-DOS/Windows users with fewer alternatives.

Those packages that integrate the VCR control and database services into one program tended to provide either free-format data (where the user can type any length or content of notes desired) or flat-file data (where the user has to limit coding to a fixed number of predetermined codes). Free-format data files provide the user ultimate flexibility in coding, but they involve increased data storage requirements and decreased data retrieval speed. Flat-file technologies reduce storage needs and increase the speed of access but restrict the user to a finite list of allowable codes. Using criteria such as those we have described allows potential users to view each program's particular requirements as a benefit or a restriction, depending on the specific research project and its coding needs.

The Technological Innovations in Educational Research (TIER) laboratory engages in a number of research projects aimed at improving the use of technology in education and educational research. Many of these projects have been using video as one means of data collection, employing varying methods for understanding the events recorded on the videotape. An increasing number and intensity of these projects led us to consider computerassisted means for data coding and analysis. Our situation required us to make use of MS-DOS/Windows compatible computers and the video camcorders and VCRs available at our university. These research projects involved 
numerous interacting variables of interest, requiring whatever analytical tool we used to be able to handle a large number of potential codes in many different combinations. Our requirements also called for this tool to provide the computer-VCR interface and control; quick database services, including the ability to easily change previously coded events; basic reporting capabilities, including the ability to easily transfer data to and from other programs that might be used for further analyses; and, because of the limited number of VCRs available, the ability to simulate a VCR on another computer for offline data examination. Unfortunately, none of the available software packages provided this combination of features and flexibility in function. Therefore, in 1992 we undertook a development effort to address these needs and create a research tool that would be of use in our laboratory's video research.

\section{THE VTLOGANL DEVELOPMENT PROJECT}

We began with the premise that transcript and written description are, in many studies, an unnecessary complication of the analytical process. Although the written word remains necessary for communicating the results of research, the process of transforming sights and sounds into text and then analyzing the text for conceptual insight is both time-consuming and subject to inaccuracy.

We also accepted a hierarchical schema for describing the kinds of events to be coded. At the bottom of this structure is the pulsed event, where only the simple occurrence of a behavior or expression is of interest. The researcher may be studying when these events occur, how much time elapses between events, and the ordering and/or simultaneity of different events under study. Next is the duration event, when the lifespan of an event, including its time of origin and completion, is under observation. In addition to the kinds of analyses done on pulsed events, studies of duration events should allow for the computing of basic descriptive and relational statistics concerning the span of the event, the interval between events, and event overlap and periodicity. At the top of the hierarchy is the intensity event, where some quality of the event is dynamically measured and recorded. Intensity studies seek understanding of the degree of expression in addition to its duration.

The VTLOGANL project has been developed using a staged strategy. In the first stage of development (Version 1), VTLOGANL employed a very rudimentary computer control of the video deck to provide basic pulsed event coding and analysis. In Stage 2 of development, the interface between the computer and the video deck was refined, a more flexible data system was introduced, and the capacity for coding duration events was added. The Stage 3 effort (currently under way) is focusing on implementing a strategy for coding and analyzing intensity events employing the principles of fuzzy logic (Kosko, 1993; Manton, Woodbury, \& Tolley, 1994) for a nonboolean type of data analysis.
The current version of VTLOGANL (Version 2.81) is designed to be used on an MS-DOS (Version 6 or higher) computer system. The minimum hardware requirements include a 386/25-SX (or faster) processor, at least $3 \mathrm{Mb}$ of memory (with at least $2 \mathrm{Mb}$ configurable as expanded memory), at least $3.5 \mathrm{Mb}$ of hard disk space (approximately $1.5 \mathrm{Mb}$ for the program and supporting files and the remainder for data and work files), an EGA (or VGA) color (or monochrome) monitor, and a serial port (COM1 or COM2) for communicating with the video deck. A mouse is supported but is not required. VTLOGANL can control selected video tape decks through the standard RS-232 serial port on the computer. To date, the TIER lab has tested and used the Panasonic AG-1960/RS and the Panasonic AG-5700 video decks. ${ }^{1}$ With the appropriate communication cable, VTLOGANL can also interface with video camcorders and VCRs using the SONY Control-L (or LANC) communications protocols. ${ }^{2}$ This option is not as fully developed as are the Panasonic interfaces, however, and may not work correctly with all equipment or under all circumstances.

\section{VTLOGANL Terminology}

Four basic data elements are defined within the VTLOGANL system: project, tape, codeword, and logged event. VTLOGANL creates a separate data file for each and every project defined. The name of the project data file, in addition to a longer project description, help to identify the project and keep it separate from other project files. The number of projects a researcher can create is limited only by the amount of disk storage available on the computer system. All of the information about the project, including defined tapes and logged events, is stored in this single data file, allowing for easy backup and file transfer.

Before doing any logging of recorded events, the researcher must define the videotape to be logged to VTLOGANL. Each reel of videotape is given a unique tape number (1-999). Descriptive information about the events recorded on that videotape can be stored as part of the tape descriptor (see Table 1 for individual data field information). VTLOGANL also requires the researcher to declare the events to be coded on each tape as either free events or fixed events. A free events tape allows events to be coded starting from any time index and ending at any time index on the tape. Occasionally a re-

Table 1

Tape Descriptor Fields

\begin{tabular}{ll}
\hline Field Name & \multicolumn{1}{c}{ Description } \\
\hline Number & A unique number for each reel, from 1 to 999 \\
Description & A short description of what is recorded on that reel \\
Duration & 0 for "free-coded" tapes, set duration for "fixed-coded" \\
Period & 0 for "free-coded" tapes, set period for "fixed-coded" \\
Scene Date & The date the scene was taped \\
Scene Time & The time of day the scene was taped \\
Scene By & Initials of the person doing the videotaping \\
Scene Start & The time index on the tape of the start of this scene \\
Scene Stop & The time index on the tape of the end of this scene \\
Scene Note & A short description of what is recorded in this scene
\end{tabular}


search design calls for observing for only a certain amount of time every so many periods. Fixed event tapes allow the researcher to declare the span of coding interval and its period (for example, observing $30 \mathrm{sec}$ every $5 \mathrm{~min}$ ). VTLOGANL can force logged codes in a fixed-event tape to adhere to the interval and period defined for the fixed-period observations.

Within each tape, information is expressed through the use of codewords. A codeword is an eight-character (maximum) shorthand for the conceptual meaning attached to a particular event observed and/or heard on a videotape record. VTLOGANL allows a maximum of 9,999 codewords in any single project file (see Table 2 for individual data field information). In addition to the codeword name and description, the researcher can designate a default duration of each codeword when that codeword is applied to an observed event. A duration of 00:00:01 (1 sec), the shortest period of time that can be logged, describes a codeword representing a pulsed event. Duration events, on the other hand, can have either a specified duration of any fixed amount of time (according to the research design) or a duration of 00:00:00 (0 sec). VTLOGANL interprets a 0-sec duration codeword as one for which the researcher will manually indicate a starting and stopping time each time the codeword is used. Direct support for intensity events is not possible in the current version of VTLOGANL, although the researcher can simulate intensity coding by creating discrete codewords for certain predetermined levels along an intensity continuum (e.g., having different codewords for a $10 \%$ saturation, $20 \%, 30 \%$, and so on). By using the same slot for each of the different codewords (the slot is the physical location of a codeword on the logging screen), the user can ensure that only one of the pseudointensity codewords will be assigned to an event at each moment in time. In addition, an outline number may be given to each codeword. The outline number is useful for organizing the presentation of codewords according to a larger, thematic structure. This is a necessary part of the process of reconceptualizing and codeword hierarchy construction when developing meaning in a qualitative interpretation application.

Before logging any videotaped events, the researcher may elect to speed the process of codeword identification by assigning certain codewords to quick keys. A quick key is a two-key combination (e.g., the $<$ ALT $>$ key and a character key) that serves as a shorthand for the desired codewords. VTLOGANL allows the researcher to assign up to 45 different codewords to quick keys.

Table 2

Codeword Descriptor Fields

\begin{tabular}{ll}
\hline Field Name & \multicolumn{1}{c}{ Description } \\
Codeword & An eight-character alphanumeric, 9,999 maximum \\
Outline No. & A six-digit value, used to organize codewords \\
Description & A short description of the meaning of the codeword \\
Default Time & 0 if unlimited, 1 (sec) for pulsed or other events \\
Default Slot & From 1 to 30, representing the logging screen location \\
\hline
\end{tabular}

Events are logged through a graphical logging screen. This screen may be accessed in either actual mode (with a VCR slaved to the computer system) or in virtual mode (with the computer system's internal clock simulating VCR playback). The bottom portion of the logging window presents the researcher with graphic controls (accessed through either the computer's mouse or selected function keys) for controlling the operation of either the actual or virtual VCR. When VTLOGANL is used in actual mode, the program queries the VCR up to 30 times each second, reporting the actual VCR status and current time position back to the program and user. In virtual mode, VTLOGANL provides all of the same logging and editing functions, including controls for the (simulated) VCR. This allows data queries and changes to take place on another computer system-a useful feature when VCR equipment is at a premium!

VTLOGANL uses neither a flat-file nor a free-format database design; rather, codewords and the coded events are maintained in an innovative multiply indexed dynamic data file. VTLOGANL users may attach $(\log )$ up to 30 different codewords, from the maximum of 9,999 codewords that can be defined in a single project, to each elapsed second of tape time. It is conceivable, although impractical in most real cases, for each second to have a completely different set of codewords. However, even in the worst case scenario (where the maximum of 9,999 codewords is defined and all 30 logged codewords are changing every second), VTLOGANL's database implementation allows it to maintain codeword retrieval and display synchronous with realtime VCR playback!

The user organizes these multiple and potentially changing combinations of codewords by displaying each logged codeword in a particular slot on the logging window along with the starting and stopping time of that codeword's current duration. During logging, VTLOGANL will place new codewords in the first available slot, or the researcher can designate certain codewords to appear only in certain slot locations. In this way the logging screen can be ordered with codewords grouped according to the major themes being logged.

\section{An Example}

The following example, constructed from actual studies conducted in the TIER lab, illustrates the power of VTLOGANL in the coding of video records. In this example, a researcher is helping a new teacher to understand his activities while in front of his class. The researcher and the teacher have previously agreed that three aspects of the teacher's behavior would be of initial importance: speech (including talking and listening), movement around the classroom, and physical activity (such as touching or gesturing). It was further decided that how long (both total and on average) the teacher tended to spend in each of the different kinds of speech and movement activities would be of interest, whereas only the number of times each physical activity took place (not the duration of the activity) was important. An 
Table 3

Codeword List Report

\begin{tabular}{lcllcc}
\hline Codeword & Outline & \multicolumn{1}{c}{ Description } & Duration & Slot \\
\cline { 1 - 3 } LISTEN & 100 & Teacher is listening & & $::$ & 1 \\
QUESTION & 100 & Teacher is questioning & & $::$ & 1 \\
TALKING & 100 & Teacher is talking & & $::$ & 1 \\
STAND & 200 & Teacher is standing still & & $::$ & 11 \\
WALK & 200 & Teacher is walking & $::$ & 11 \\
GESTURES & 300 & Teacher gestures to an object & $:: 1$ & 21 \\
OBJECT & 300 & Teacher touches an object & $:: 1$ & 21 \\
POINTS & 300 & Teacher points to a student & $:: 1$ & 21 \\
TOUCHES & 300 & Teacher touches a student & $:: 1$ & 21 \\
\hline
\end{tabular}

initial set of nine codewords-four pulsed (for the different kinds of physical activity) and five duration (for the speech and movement) - were developed, with the understanding that new codewords might be added, or existing codewords refined, as events were observed and categorized. A project file (named CLASSOBS.VTL) was created and the initial set of nine codewords entered into the database prior to coding. Table 3 illustrates a VTLOGANL report of the initial codewords, including their outline numbers, descriptions, durations, and their preassigned slot locations.
The videotape of this teacher was then reviewed and coded. Figure 1 shows a snapshot of the logging screen at $1 \mathrm{~min}, 25 \mathrm{sec}$ into the tape. At this point, the teacher has been observed to be talking to the class, walking around the room, and pointing to a student. The duration of the talking and walking events can be clearly seen (the pointing event, being a pulsed event, has a duration of only $1 \mathrm{sec}$ ). The placement of each codeword into groups according to slot location (speech events in Slot 1, movement events in Slot 11 , and physical activity events in Slot 21) further helps the researcher to thematically organize the coding task during video review. As more codewords are developed, they can be added dynamically during the logging session without the need to exit and then reenter the logging task. For example, the topic of the teacher's speech was not initially thought to be important, but it can be easily added later by merely reviewing the tape, creating new codewords, and assigning these codewords to specific event times on the tape. Such new codewords would not have to replace the previously coded entries but instead could be assigned to new slot locations (in this case, perhaps to Slot 2 , directly underneath the more general speech codeword slot on the logging screen).

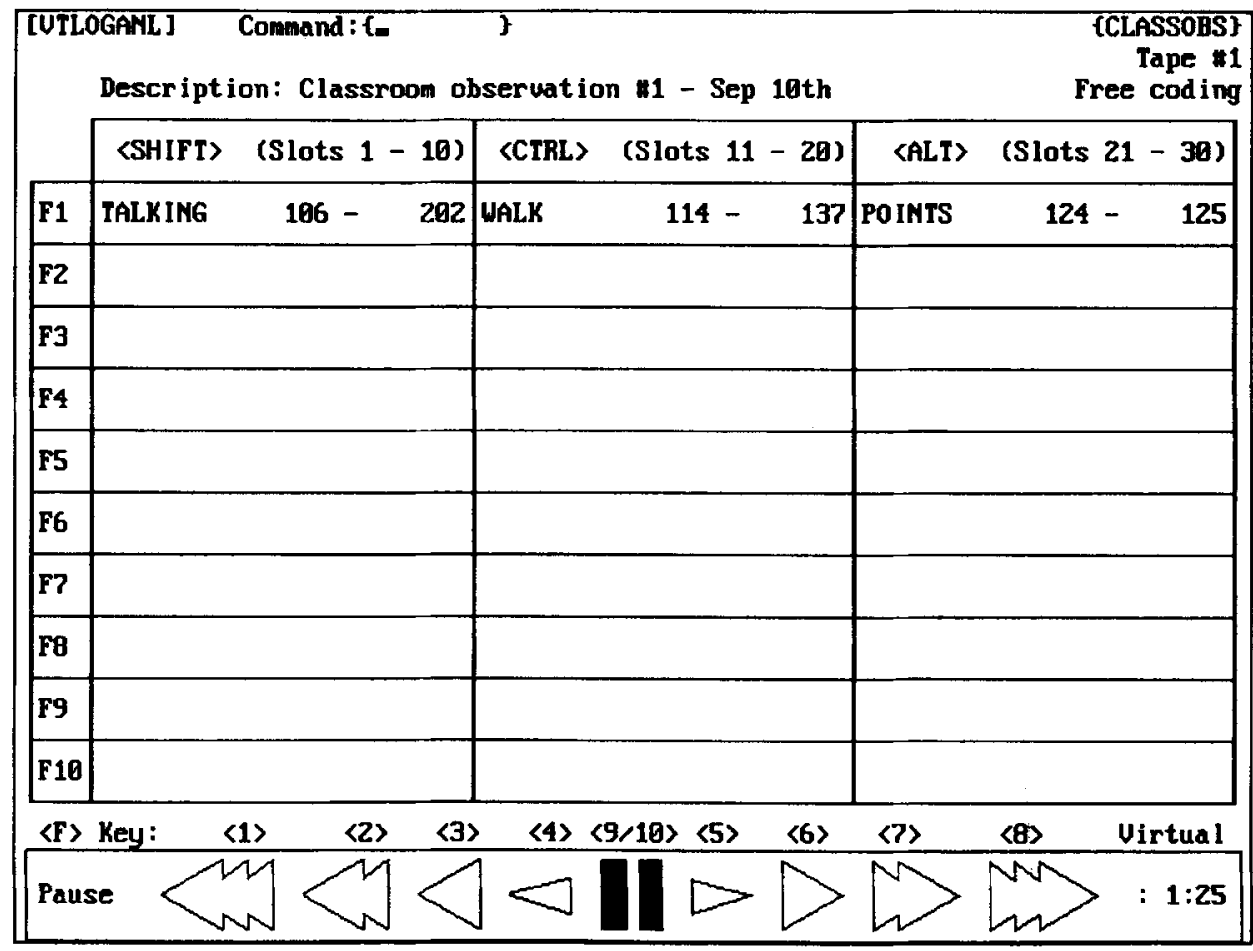

Figure 1. The VTLOGANL logging screen. Logging commands are entered in the upper left-hand portion of the screen, with description information about the tape being logged immediately below and to the right. Logged codewords, along with their starting and ending times, are displayed in the slots numbered 1-30. Open duration codes may be ended by the user by touching the $<$ SHIFT $>,\langle$ CTRL $>$, or $<$ ALT $>$ key and the appropriate function key $(<F 1>$ through $<F 10>$ ) corresponding to that slot. Video deck control buttons are along the bottom row, and may be accessed either through the mouse pointer or by the appropriate function key. Deck status is displayed in words on the bottom left, and tape index time is displayed on the bottom right. Also along the bottom right is an indicator of whether VTLOGANL is operating in "Actual" (real tape deck connected) or "Virtual" (simulated tape deck) mode. 


\section{Features Summary}

VTLOGANL Version 2.81 is an MS-DOS based, menudriven program using both keyboard and, if desired, mouse inputs. Researchers with limited computer experience will find the VTLOGANL interface quick to learn and easy to master. VTLOGANL works with both relatively inexpensive yet accurate "pro-sumer" camcorders and VCRs without the need for special equipment, interfaces, or the sacrifice of one (or more) audio tracks. VTLOGANL's database design allows researchers to enter and display, in realtime, up to 30 different codewords (drawn from a pool of up to 9,999) for each second of elapsed tape time. Codewords can be represented as either pulsed or duration events, or can simulate intensity events. Further, users can elect to freely enter codewords whenever events are observed or to maintain fixed observation periods and intervals.

By using the computer system's internal clock to simulate a VCR (when in virtual mode), researchers can revisit the logging task without needing to run a real VCR. This feature permits an off-line examination of logged events and allows multiple researchers to work on different aspects of the same project simultaneously when video equipment is limited but a second computer system is available.

VTLOGANL allows for the printed reporting of all data elements in the project file. Codewords can be reported in either alphabetical or outline number order, and several options exist for aggregating and reporting logged events (including the calculation of basic descriptive statistics). All elements can also be selected to report to an ASCII text file for those researchers desiring to move data into another software package for a more detailed or statistically intensive analysis or to combine VTLOGANL data with data from other sources. VTLOGANL can also automatically select segments of video previously identified by the researcher as exemplars and then produce an instruction file (called an EDL) to computer-controlled video editing equipment to copy these segments to duplicate tapes. This feature is invaluable to researchers who have access to computer-controlled video editing equipment when they are preparing a video presentation using selected cuts from raw data.

The automatic renaming and collapsing of multiple codewords into one codeword is a critical feature for researchers using VTLOGANL in a grounded theory analysis. This capability can be used to rename codewords that might have originally been given confusing labels. Several different codewords can also be collapsed into a single codeword when duplicate or overlapping meanings are discovered. Using each codeword's outline number, together with outline-ordered printed reports, helps researchers develop patterns and structures of codewords that can be used to identify these irregularities.

Finally, VTLOGANL is flexible enough to allow several researchers to work on the same project's videotapes within the same data file. Researchers may elect to share the same codewords while independently coding the same physical tapes, or may develop their own codeword structures for independent coding. VTLOGANL can then report these codings separately or in combination, allowing for a quick comparison of interrater reliability and coding structure.

\section{CONCLUSION}

VTLOGANL has already been instrumental to the implementation of several research efforts, including Project Homeroom and Project FIRST (Hecht, Dwyer, Roberts, Schoon, \& Fansler, 1994; Hecht, Roberts, Dwyer, Schoon, \& Fansler, 1994). The VTLOGANL method is also gaining wider use as it is disseminated at regional and national meetings (Hecht, Roberts, Dwyer, \& Schoon, 1994; Roberts, Hecht, Dwyer, \& Schoon, 1993).

Further development is continuing on the VTLOGANL project, with current work focusing on adding the capacity for coding intensity events as part of the Stage 3 design effort. In addition to traditional boolean logic queries, VTLOGANL is being developed to include an analytical system of multivalued logics that would be more applicable to understanding intensity-coded events. Although the application of fuzzy logic, as it is more commonly known, has become well known in the areas of process control and manufacturing, it has been somewhat slower to gain widespread use in the social sciences (Wang, 1983; Wang \& Chang, 1980). An increasing number of studies, however, validate the utility of reasoning through a system of multivalued logics in economics, psychology, sociology, and education (Kay \& McDaniel, 1978; Oden, 1977; Pfeilsticker; 1981; Zimmerman, 1985). We feel that this continued development is important, since the use of videotape will remain somewhat suspect until more systematic, refined, and tested methods are in use by the research community.

\section{Availability}

A free copy of the VTLOGANL software may be obtained by either sending an MS-DOS formatted, high density diskette and SASE to the authors, or by electronic file transfer (anonymous FTP) through the Internet from the tierlablvtloganl directory at the ftp.iLstu. edu computer system.

\section{REFERENCES}

Barba, R. H., \& Rubba, P. A. (1992). Procedural task analysis: A tool for science deduction problem solving research. School Science \& Mathematics, 92, 188-192.

Carter, K., \& ANDERSon, B. (1989). Can video research escape the technology? Some reflections on the problems and possibilities of A.V. research. SIGCHI Bulletin, 21, 112-114.

Gardner, C. H., Clements, R. O., \& Rodriquez, M. (1982). Comparing videotapes and written narrative records of second grade reading classes: Some preliminary findings. Austin: University of Texas, Research and Development Center for Teacher Education. (ERIC Document Reproduction Service No. ED 215 332)

HeCht, J. B., DWyer, D. J., Roberts, N. K., Schoon, P. L., \& FANSLER, G. (1994). Project Homeroom, Project Schoolroom, and Regular School: Innovations in team teaching, interdisciplinary learning, and the use of technology. Normal: Illinois State University, Technological Innovations in Educational Research Laboratory. 
Hecht, J. B., Roberts, N. K., DWyer, D. J., SChoOn, P. L., \& FANSLER, G. (1994). Project FI.R.S.T. First year impressions. Normal: Illinois State Unıversity, Technological Innovations in Educational Research Laboratory.

Hecht, J. B., Roberts, N. K., DWyer, D. J., \& SChoon, P. L. (1994, April). VTLOGANL A computer program for coding and analyzing data gathered on video tape. Paper and computer software demonstration presented at the 1994 annual meetıng of the American Educational Research Association, New Orleans

KAY, P., \& MCDANIEL, C. (1978). The linguistic significance of the meanings of basic color terms. Language, 54, 61-64.

KIEREN, D. K., \& MUNRo, B. (1985). The observational recording dilemma. Ottawa, ON: Social Sciences and Humanitıes Research Council of Canada. (ERIC Document Reproduction Service No. ED $297021)$

KNAPP, M. L., \& HARRISON, R. P. (1972). Observing and recording the nonverbal data in human transaction. Paper presented at the annual meeting of the Speech Communication Association, Chicago. (ERIC Document Reproduction Service No. ED 074 528)

Kosko, B. (1993). Fuzzy thinking. The new science of fuzzy logic. New York: Hyperion.

Krauss, R. M., Morrel-Samuels, P., \& HochberG, J. (1988).Videologger: A computerized multichannel event recorder for analyzing videotapes. Behavior Research Methods, Instruments, \& Computers, 20, 37-40.

Lo, J., Wheatley, G. H., \& Smith, A. C. (1991). Learning to talk mathematics. Paper presented at the annual meeting of the American Educational Research Association, Chicago. (ERIC Document Reproduction Service No. ED 334 073)

Manton, K. G., Woodbury, M. A., \& Tolley, H. D. (1994). Statistical applications using fuzzy sets. New York: Wiley.

MCKAY, W. E. (1989). EVA. An experimental video annotator for symbolic analysis of video data. SIGCHI Bulletin, 21, 68-71.

NEAL, L. (1989). The use of video in empirical research. SIGCHI Bulletin, 21, 100-102.

ODEN, G. C. (1977). Fuzziness in semantic memory: Choosing exemplars of subjective categories. Memory \& Cognition, 5, 198-204.

Packer, M. J., \& Richardson, E. (1989, April). Strategies of social entry among preschool children. Paper presented at the biennial meeting of the Society for Research in Child Development, Kansas City, KS. (ERIC Document Reproduction Service No. ED 312 031)

PFEILSTICKER, A. (1981). The systems approach and fuzzy set theory: Bridging the gap between mathematical and language oriented economists. Fuzzy Sets \& Systems, 6, 209-233.
Roberts, N. K., HeCht, J. B., DWyer, D. J., \& SCHOON, P. L. (1993, October). VTLOGANL: A methodology for the qualitative analysis of videotaped data. Paper and workshop presented at the annual meeting of the Midwestern Educational Research Association, Chicago.

Roschelle, J., \& Goldman, S. (1991). VideoNoter: A productivity tool for video data analysis. Behavior Research Methods, Instruments, \& Computers, 23, 219-224.

SCARSELletTA, D. M. (1987). Observation of children's social play: The basis for a system of coding. Unpublished master's thesis, State University of New York. (ERIC Document Reproduction Service No. 292 564)

SCHOTSBERGer, P. G. (1993, April). An approach to analyzing the videotaped problem solving behaviors of individual students. Paper presented at the annual meeting of the American Educational Research Association, Atlanta.

Stallings, J. A., \& Giesen, P. A. (1977, April). The study of reliability in observational data. Paper presented at the annual meeting of the American Educational Research Association, Chicago.

TAIT, D. M. (1993). Video Analysis, a method of assessing changes in preverbal and early linguistic communication after cochlear implantation. Ear \& Hearing, 14, 378-389

WANG, P. W. (1983). Advances in fuzzy sets, possibility theory, and applications. New York: Plenum.

Wang, P. W., \& Chang, S. K. (1980). Fuzzy sets Theory and applications to policy analysis and information systems. New York Plenum.

ZimmermanN, H. J. (1985). Fuzzy set theory and its applications. Boston: Kluwer.

\section{NOTES}

1. The Panasonic AG-1960/RS is a high-end consumer S-VHS, serialcontrollable video deck with a built-1n receiver and tuner. It retails for about $\$ 1,300$. The Panasonic AG-5700 is an industrial S-VHS, serialcontrollable video deck without a receiver or tuner, retailıng for about $\$ 1,100$.

2. Many SONY (and third-party compatible) video products are designed to work with the Control-L (or LANC) interface. A connecting cable converting Control-L signals to standard RS-232 can be obtained for under $\$ 100$

(Manuscript received October 3, 1994; revision accepted for publication February 28, 1995.) 